\title{
Reciprocity Relation between the Mass Constituents of the Universe and Hardy's Quantum Entanglement Probability
}

\author{
Hans Hermann Otto \\ Materials Science and Crystallography, Technical University of Clausthal, Clausthal-Zellerfeld, Lower Saxony, Germany \\ Email: hhermann.otto@web.de
}

How to cite this paper: Otto, H.H. (2018) Reciprocity Relation between the Mass Constituents of the Universe and Hardy's Quantum Entanglement Probability. World Journal of Condensed Matter Physics, 8, 30-35.

https://doi.org/10.4236/wjcmp.2018.82003

Received: April 18, 2018

Accepted: May 14, 2018

Published: May 17, 2018

Copyright $\odot 2018$ by author and Scientific Research Publishing Inc. This work is licensed under the Creative Commons Attribution International License (CC BY 4.0).

http://creativecommons.org/licenses/by/4.0/

\begin{abstract}
In this short contribution, a reciprocity relation between mass constituents of the universe was explained governed by Hardy's maximum entanglement probability of $\varphi^{5}=0.09017$. While well explainable through a set-theoretical argumentation, the relation may also be a consequence of a coupling factor attributed to the normed dimensions of the universe. Also, very simple expressions for the mass amounts were obtained, when replacing the Golden Mean $\varphi$ by the Archimedes constant $\pi$. A brief statement was devoted to the similarity between the E-Infinity Theory of El Naschie and the Information Relativity Theory of Suleiman. In addition, superconductivity was also linked with Hardy's entanglement probability.
\end{abstract}

\section{Keywords}

Universe, Mass Constituents, Golden Mean, Archimedes' Constant, Reciprocity Relation, E-Infinity Theory, Information Relativity Theory, Entanglement, Superconductivity

\section{Introduction}

Since Mermin [1] recasted the results of Hardy's Gedankenexperiment [2] of entangled states for two quantum particles with the maximum nonlocal effect in the form of a power of the Golden Mean $\varphi=\frac{1}{2}(\sqrt{5}-1)$ giving

$$
P=\gamma_{\max }=\frac{1}{2}(5 \sqrt{5}-11)=5 \varphi-3=\varphi^{5}=0.090169943 \cdots,
$$

this quantum entanglement probability $P$ was shown by the outstanding Egyptian physicist El Naschie to describe the many puzzling features of our universe 
very well such as dark energy, negative gravity or accelerated expansion of the universe [3].

In this short contribution, I want to complete a previous publication [4] and will describe the mass respectively energy constituents of our universe solely by Hardy's probability of quantum entanglement of two quantum particles, pointing to an unexpected reciprocity relation between mass constituents of the universe.

Besides the E-infinity theory of El Naschie [5], the recently developed Information Relativity Theory (IR), created by Suleiman [6], should attract our attention, too. This theory offers a new physical interpretation of the dynamics of matter-wave duality confirming the de Broglie-Bohm interpretation of the Quantum Theory [7] [8] [9]. The chosen time respectively length transformations automatically involve the Golden Mean, which is the basic building unit of the $E$-infinity theory. According to the $I R$ approach, the maximum kinetic energy density of a moving body at a recession velocity $\beta=v / c=\varphi$ is derived being $\varphi^{5}[6]$

$$
\frac{e_{m}}{e_{o}}=\frac{1-\beta}{1+\beta} \beta^{2}=\frac{1-\varphi}{1+\varphi} \varphi^{2}=\varphi^{5}, \text { using } \beta=\varphi
$$

Indeed, this transformation equation for the kinetic energy density in terms of the recession velocity $\beta$ resembles the maximum quantum entanglement probability $P$ of two quantum particles given by Hardy [2] respectively Mermin [10]

$$
P(2 G, 2 G)=\frac{1-x}{1+x} x^{2} \quad[2][10] .
$$

Both mentioned theories ( $E$ respectively $I R$ ), if they can be brought into line, in the end, will have a lasting effect on our thinking and the perception of our existence.

\section{Results}

\subsection{Reciprocity Relation and Hardy's Entanglement Probability}

According to given results of the set-theoretical approach of $E$-infinity describing the five-dimensional Kaluza-Klein spacetime [4] [11], the amount of baryonic matter of the universe emerges as

$$
\left.\Omega_{b}=\frac{1}{2} \varphi^{5}=0.04508 \ldots \quad \text { (about } 4.51 \%\right) .
$$

The dark matter amount can be recast in the very simple form of

$$
\left.\Omega_{d}=\frac{1}{100} 2 \varphi^{-5}=0.22180 \ldots \quad \text { (about } 22.18 \%\right) .
$$

It may not be pure fortuity but nevertheless surprising that both amounts show a reciprocity relation. This can be seen, if we write down the remaining dark energy amount as the difference to the entire mass in a more persuasive form 


$$
\Omega_{\Lambda}=1-\frac{1}{10}\left(5 \varphi^{5}+\left(5 \varphi^{5}\right)^{-1}\right) \quad(\text { about } 73.31 \%)
$$

Such coincidence means that both mass constituents should not be considered independent of each other. Mathematically, reciprocity is found, for instance, if one considers volume in comparison to surface, or particles in comparison to waves. Relevant topological arguments from the set theory are summarized by $E I$ Naschie [11]. However, the denominator factor of 100 in Equation (5) may be interpreted as a coupling term equal to the normed dimensions of the universe [12].

The entire dark constituents yields

$$
\Omega_{d+\Lambda}=\frac{5}{2} \varphi^{2}=0.954915 \ldots
$$

By the way, the simple result of $5 \varphi^{2}$ represents the five-dimensional surface of the pre-quantum wave being the cobordism of the topological volume of $\varphi^{5}$ of the Kaluza-Klein five-dimensional manifold [4] [13] [14].

The estimated constituents are fairly well consistent with measurements of the Wilkinson Microwave Anisotropy Probe mission ( WMAP) [15].

El Naschie recently pointed out that $D=\varphi^{-5}=11+0.0901699 \ldots$ obviously represents the fractal dimension $D_{\mathrm{M}}$ of Witten's $M$-theory [16] [17].

Because $\varphi^{-5}=11+\varphi^{5}$, its beautiful hierarchical form (or continued fraction representation) can be expressed as

$$
\begin{aligned}
\varphi^{-5} & =11+\frac{1}{11+\frac{1}{11+\frac{1}{11+\ldots .}}} \\
& =11+0.0901699 \ldots,
\end{aligned}
$$

in contrast to the most unique representation for the Golden mean

$$
\begin{aligned}
\varphi & =\frac{\sqrt{5}-1}{2}=\frac{1}{1+\frac{1}{1+\frac{1}{1+\ldots .}}} \\
& =0.618033989 \ldots
\end{aligned}
$$

\subsection{Alternative Approach Using Archimedes' Constant $\pi$}

An alternative approach for the mass constituents of the universe by applying the Archimedes constant $\pi$ instead of $\varphi$ yields almost equal amounts in comparison to results according to Equations (4) to (7) [18] [19]

$$
\begin{gathered}
\Omega_{b}=\frac{\pi-3}{\pi}=0.0450703 \ldots, \\
\Omega_{d}=\frac{\pi}{(\pi-3) 100}=0.221875 \ldots, \\
\Omega_{\Lambda}=1-\frac{1}{10}\left(\frac{10(\pi-3)}{\pi}+\frac{\pi}{10(\pi-3)}\right)=0.733054 \ldots,
\end{gathered}
$$


and finally

$$
\Omega_{d+\Lambda}=\frac{3}{\pi}=0.9549296 \ldots
$$

for the sum of the dark constituents (matter and energy). It may turn out in the future whether this replacement may be of any physical importance. However, some numerical approximations connect $H a r d y^{2}$ s entanglement probability $\varphi^{5}$ with Archimedes constant $\pi$ and the inverse of Sommerfeld's fine structure constant $\bar{\alpha}_{0}$, respectively [20]

$$
\begin{aligned}
& \pi-3 \approx \frac{16}{137-24}=0.14159292 \\
& \bar{\alpha}_{0} \approx 137+\frac{2}{5} \varphi^{5}=137.03606 \ldots
\end{aligned}
$$

\subsection{Hardy's Entanglement Probability and Superconductivity}

If one deals with particle entanglement, superconductivity represents a physical phenomenon suspecting such property. Some years ago, the present author suggested linking the optimum hole doping $\sigma_{\mathrm{o}}$ of high- $T_{\mathrm{c}}$ superconductors with Hardy's $\varphi^{5}$ entanglement probability [18]. The fractal-hierarchical structure of electrons entangled in pairs obviously determines this optimum near a quantum critical point that can be linked on the one hand with the universal fractal constant $\delta_{1}=8.7210972 \ldots$ of the renormalized quadratic Hénon map (remember the quadrilateral layer structure of the cuprates)

$$
\sigma_{o} \approx \frac{2}{\delta_{1}}=0.2293
$$

on the other hand with $H a r d y^{\prime}$ s entanglement probability

$$
\sigma_{o} \approx \frac{8}{\pi} \varphi^{5}=0.2296
$$

this time connecting $\varphi$ and $\pi$, the most important universal numbers of the cosmos.

The quotient of the Fermi speed to the Klitzing speed would yield

$$
v_{F} / v_{K} \approx \frac{2}{\pi} \varphi^{5}=0.0571
$$

which is again proportional to $\varphi^{5}$ [20].

The fractal nature of electronic response in superconductors was documented some years ago by scanning tunneling microscopy [21] [22] and is consistent with generated $d$-wave fractal patterns in superconductors as a consequence of antiferromagnetism [23].

\section{Conclusion}

This contribution points to a reciprocity relation between the mass constituents of the universe that suggests their common physical interrelation. The obtained baryonic respectively dark mass amounts are related to Hardy's maximum par- 
ticle entanglement probability, namely the fifth power of the Golden Mean. Importantly, it is recommended once more to replace the Golden Mean $\varphi$ in the mass relations by the Archimedes constant $\pi$ to utilize very simple expressions that await further interpretation. The competition between the two numbers obviously determines what the realities of our universe are. However, a Nature article just published reporting on a galaxy lacking dark matter halo is reason enough to re-evaluate the statements given here [24]. The opportunity opens to do an acid test with Suleiman's theory [6]. The author's real interest in the cosmological relations including entanglement considerations is to catch an idea about the possible dark side of superconductivity.

\section{References}

[1] Mermin, N.D. (1994) Quantum Mysteries Refined. American Journal of Physics, 62, 880-887. https://doi.org/10.1119/1.17733

[2] Hardy, L. (1993) Nonlocality for Two Particles without Inequalities for Almost All Entangled States. Physical Review Letters, 71, 1665-1668.

https://doi.org/10.1103/PhysRevLett.71.1665

[3] El Naschie, M.S. (2013) Quantum Entanglement: Where Dark Energy and Negative Gravity plus Accelerated Expansion of the Universe Comes from. Journal of Quantum Information Science, 3, 57-77. https://doi.org/10.4236/jqis.2013.32011

[4] Otto, H.H. (2018) Mass Constituents of a Flat Lattice Multiverse: Conclusion from Similarity between Two Universal Numbers, the Rocksalt-Type 2D Madelung Constant and the Golden Mean. Journal of Modern Physics, 9, 1-13. https://doi.org/10.4236/jmp.2018.91001

[5] El Naschie, M.S. (2004) A Review of E-Infinity and the Mass Spectrum of High Energy Particle Physics. Chaos, Solitons \& Fractals, 19, 209-236. https://doi.org/10.1016/S0960-0779(03)00278-9

[6] Suleiman, R. (2017) Dark Matter and Dark Energy as Quantum Entities. Researchgate. Working Paper, 1-12.

[7] De Broglie, L. (1923) Waves and Quanta. Nature, 112, 540. https://doi.org/10.1038/112540a0

[8] Bohm, D. (1952) A Suggested Interpretation of the Quantum Theory in Terms of "Hidden" Variables. I. Physical Review, 85, 166-179. https://doi.org/10.1103/PhysRev.85.166

[9] Bohm, D. (1952) A Suggested Interpretation of the Quantum Theory in Terms of "Hidden" Variables. II. Physical Review, 85, 180-193. https://doi.org/10.1103/PhysRev.85.180

[10] Mermin, D. (1998) The Ithaca Interpretation of Quantum Mechanics. Pramana, 51, 549-565. https://doi.org/10.1007/BF02827447

[11] El Naschie, M.S. (2017) From a Dual Einstein-Kaluza Spacetime to 'tHooft Renormalon and the Reality of Accelerated Cosmic Expansion. Journal of Modern Physics, 8, 1-7. https://doi.org/10.4236/jmp.2017.88085

[12] El Naschie, M.S., Olsen, S., Helal, M.A., Marek-Crnjak, L. and Nada, S. (2018) On the Missing Link between Cosmology and Biology. International Journal of Innovation in Science and Mathematics, 6, 11-13.

[13] He, J.H. (2010) A Note on Elementary Cobordism and Negative Space. Internation- 
al Journal of Nonlinear Science and Numerical Simulations, 11, 1093-1095. https://doi.org/10.1515/IJNSNS.2010.11.12.1093

[14] Marek-Crnjac, L. (2011) The Hausdorff Dimension of the Penrose Universe. Physics Research International, 2011, Article ID 874302.

https://doi.org/10.1155/2011/874302

[15] Wilkinson Microwave Anisotropy Probe 9-Year Results Released (2017) NASA Home Page.

[16] El Naschie, M.S. (2018) Resolution of Hardy's Paradox within Spacetime Physics and the Ithaca Interpretation of Quantum Mechanics. World Journal of Condensed Matter Physics, 8, 23-29. https://doi.org/10.4236/wjcmp.2018.82002

[17] Witten, E. (1995) String Theory Dynamics in Various Dimensions. Nuclear Physics $B$, 443, 85-126. https://doi.org/10.1016/0550-3213(95)00158-O

[18] Otto, H.H. (2017) Should We Pay More Attention to the Relationship between the Golden Mean and the Archimedes' Constant? Nonlinear Science Letters A, 8, 410-412.

[19] Otto, H.H. (2017) Gyromagnetic Factor of the Free Electron: Quantum-Electrodynamical Correction Expressed Solely by the Golden Mean. Nonlinear Science Letters A, 8, 413-415.

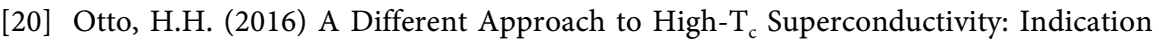
of Filamentary-Chaotic Conductance and Possible Routes to Superconductivity above Room Temperature. World Journal of Condensed Matter Physics, 6, 244-260. https://doi.org/10.4236/wjcmp.2016.63023

[21] Kohsaka, Y., Taylor, C., Fujita, K., Schmidt, A., Lupien, C., Hanaguri, T., Azuma, M., Takano, M., Eisaki, H., Takagi, H., Uchida, S. and Davis, J.C. (2007) An Intrinsic Bond-Centered Electronic Glass with Unidirectional Domains in Underdoped Cuprates. Science, 315, 1380-1385. https://doi.org/10.1126/science.1138584

[22] Phillabaum, B., Carlson, E.W. and Dahmen, K.A. (2012) Spatial Complexity Due to Bulk Electronic Nematicity in a Superconducting Underdoped Cuprate. Nature Communications, Article No. 915. https://doi.org/10.1038/ncomms1920

[23] Sachdev, S. (2014) Quantum Entanglement \& Superconductivity. Public Lecture at the Perimeter Institute, Waterloo, Ontario.

[24] Van Dokkum, P., Danieli, S., Cohen, Y., Merritt, A., Romanowsky, A.J., Abraham, R., Brodie, J., Conroy, C., Lokhorst, D., Mowla, L., O’Sullivan, E. and Zhang, J. (2018) A Galaxy Lacking Dark Matter. Nature, 555, 629-632.

https://doi.org/10.1038/nature25767 